\title{
The Evolution of Vortex Tilt and Vertical Motion of Tropical Cyclones in Directional Shear Flows
}

\author{
JIAN-FENG GU \\ Key Laboratory of Mesoscale Severe Weather, Ministry of Education, and School of Atmospheric Sciences, \\ Nanjing University, Nanjing, China, and Department of Meteorology, University of Reading, Reading, United Kingdom
}

\section{ZHE-MIN TAN}

Key Laboratory of Mesoscale Severe Weather, Ministry of Education, and School of Atmospheric Sciences, Nanjing University, Nanjing, and State Key Laboratory of Severe Weather, Chinese Academy of Meteorological Sciences, China Meteorological Administration, Beijing, China

\author{
XIN QIU \\ Key Laboratory of Mesoscale Severe Weather, Ministry of Education, and School of Atmospheric Sciences, \\ Nanjing University, Nanjing, China
}

(Manuscript received 18 January 2018, in final form 6 August 2018)

\begin{abstract}
Recent studies have demonstrated the importance of moist dynamics on the intensification variability of tropical cyclones (TCs) in directional shear flows. Here, we propose that dry dynamics can account for many aspects of the structure change of TCs in moist simulations. The change of vortex tilt with height and time essentially determines the kinematic and thermodynamic structure of TCs experiencing directional shear flows, depending on how the environmental flow rotates with height, that is, in a clockwise $(\mathrm{CW})$ or counterclockwise (CC) fashion. The vortex tilt precesses faster and is closer to the left-of-shear (with respect to the deep-layer shear) region, with a smaller magnitude at equilibrium in CW hodographs than in CC hodographs. The low-level vortex tilt and accordingly more low-level upward motions are ahead of the overall vortex tilt in CW hodographs but are behind the overall vortex tilt in CC hodographs. Such a configuration of vortex tilt in $\mathrm{CW}$ hodographs is potentially favorable for the continuous precession of convection into the upshear region but in CC hodographs it is unfavorable. Most of the upward motions within a TC undergoing CW shear are concentrated in the downshear-left region, whereas those in the $\mathrm{CC}$ shear are located in the downshear-right region. Moreover, the upward (downward) motions are in phase with positive (negative) local helicity in both $\mathrm{CW}$ and CC hodographs. Here, we present an alternative mechanism that is associated with balanced dynamics in response to vortex tilt to explain the coincidence and also the distribution variability of vertical motions, as well as local helicity in directional shear flows. The balanced dynamics could explain the overlap of positive helicity and convection in both moist simulations and observations.
\end{abstract}

\section{Introduction}

Large-scale vertical wind shear (VWS), also referred to as deep-layer shear, is commonly defined as the difference between horizontal wind vectors in the 200- and 850-hPa layers, averaged over an area in an annular region or within a given radius from the tropical cyclone (TC) center. Although deep-layer shear is responsible for TC genesis (Gray 1968; Tuleya and Kurihara 1981), structure (Black et al. 2002; Corbosiero and Molinari

Corresponding author: Zhe-Min Tan, zmtan@nju.edu.cn
2003; Reasor et al. 2013; Zhang et al. 2013; DeHart et al. 2014; Gu et al. 2016), and intensity change (Simpson and Riehl 1958; DeMaria 1996; Frank and Ritchie 2001; Wu and Braun 2004; Riemer et al. 2010; Tang and Emanuel 2010; Gu et al. 2015), it alone is not enough to represent the overall vertical structure of a large-scale environmental flow. For example, observational analysis (Wang et al. 2015) and idealized simulations (Finocchio et al. 2016) both found that low-level shear is more destructive to TC intensification than is deep-layer shear.

Most studies simplify the VWS as a unidirectional shear with the same shear direction throughout the deep 
layer. However, the profile of large-scale environmental wind can be considerably more complex in the real atmosphere. The horizontal wind may change in both magnitude and direction with height, leading to a directional shear flow that has large impacts on TC evolution. Nolan (2011, hereafter N11) first investigated the effect of a curved wind profile on TC intensification. With an identical deep-layer shear, the vortex embedded in a clockwise $(\mathrm{CW})$ hodograph significantly intensifies, whereas that in a counterclockwise (CC) hodograph does not. Onderlinde and Nolan (2014, hereafter ON14) introduced a new parameter called tropical cyclonerelative environmental helicity (TCREH) to describe such an environment. They found that positive TCREH in CW hodographs leads to the coexistence of positive local helicity and convection. The overlap of positive helicity and convection has the potential to support long-lasting thunderstorms, generating more persistent heating, thus leading to TC intensification. In contrast, the negative TCREH in CC hodographs leads to the displacement of convection from positive local helicity, is unfavorable for the maintenance of the convection required to assist intensification, and may even result in weakening of the TCs. A follow-up study (Onderlinde and Nolan 2016, hereafter ON16) attributed the various intensification rates to the position of convection, the surface latent heat flux relative to the wind shear vector, and their ability to advance into the upshear (US) region.

The findings of N11, ON14, and ON16 are based primarily on the moist dynamics that govern the interaction between convection and the TC vortex. However, moist dynamics alone cannot explain how distinct structures develop initially before the feedback (e.g., convective heating, moistening of midlevels) from convection takes effect. For example, why is convection located in the downshear-left (DSL) region of a CW hodograph but in the downshear-right (DSR) region of a CC hodograph? Why does convection usually overlap with positive local helicity in both CW and CC hodographs (Fig. 9 in ON14)? More generally, how is local helicity related to other structural changes in, for example, vortex tilt or boundary layer inflow? The fundamental dynamics underlying these features in directional shear flows have not been directly explored in previous studies. These topics will be discussed in this study.

Here, we propose that it is the dry dynamics that first regulate the different features of TCs in the directional shear flows. The view of helicity could be understood as part of dry dynamics. It has been shown that dry dynamics (Jones 1995) are essential for our understanding of the evolution of TC-like vortices in unidirectional shear flows. Therefore, a natural first step is to examine vortex evolution in directional shear flows using dry experiments that exclude feedback from moist convection. Some differences in vortex structure and tilt evolution compared with what is observed in unidirectional shear flows are expected for the following reasons. First, the direction of vertical wind shear changes with height and results in varying vortex tilts at different levels. Second, different vortex tilts will lead to distinct vortex centers at the various vertical levels, resulting in varying interactions between cyclonic circulations at different heights. Accordingly, the kinematic and thermodynamic structures will differ between $\mathrm{CW}$ and $\mathrm{CC}$ directional shear flows. An understanding of this will greatly improve our ability to predict the evolution of TC structure and intensity in directional shear flows.

The remainder of this paper is organized as follows. Section 2 describes the experimental design and the basic configuration of the numerical model. Section 3 presents the main results: section 3 a describes the evolution of vortex tilt; section $3 \mathrm{~b}$ discusses kinematic and thermodynamic structural changes and their relationship with the distribution of local helicity; and section $3 \mathrm{c}$ outlines the potential role of varying vortex tilt with height, particularly low-level vortex tilt, on the distinct evolution of TCs in pure CW and CC directional shear flows. Section 4 provides a discussion of the fundamental role of dry dynamics, explanations of vortex tilt evolution from the perspective of vortex Rossby wave dynamics, possible impact of asymmetric inflows in the boundary layer, and the generality of these results to mature storms. Finally, a brief summary is given in section 5 .

\section{Numerical model and simulation design}

The Weather Research and Forecasting (WRF) Model, version 3.4 (WRFV3.4; Skamarock et al. 2008), is used with the point-downscaling (PDS) method (N11) to investigate vortex evolution in a directional shear flow. To balance the directional flow with pressure forces, artificial forcing terms are added to the momentum equations. The absence of a temperature gradient, which would be present if pressure-forcing terms were not added to the equations, has been shown to have little impact on vortex evolution during TC genesis and also allows for doubly periodic boundary conditions (N11). The PDS method allows the background shear to be nearly constant so that TC structural and intensity changes can be primarily attributed to the directional shear.

Three domains are used in the simulations with horizontal resolutions of 9,3 , and $1 \mathrm{~km}$ and $421 \times 421,241 \times$ 241 , and $481 \times 481$ grid points, respectively. The top of the domain is $20 \mathrm{~km}$, and 41 vertical levels are used. The Coriolis parameter is set to $5.0 \times 10^{-5} \mathrm{~s}^{-1}$. No parameterizations of cumulus, microphysics, and planetary boundary layer 
processes are used because the goal is to examine the dry dynamics of vortex evolution in directional shear flows as much as possible. Dunion (2011) moist tropical sounding is used to derive the temperature in the environment. The initial vortex is a modified baroclinic Rankine vortex with a maximum tangential wind speed of $20 \mathrm{~m} \mathrm{~s}^{-1}$ and a radius of maximum wind of $90 \mathrm{~km}$. To ensure that the vortex center is calculated correctly within a range of $400 \mathrm{~km}$ (see section 3a), results are analyzed using data in the coarse-resolution domain because the vortex tilt will increase to $300-400 \mathrm{~km}$, comparable to the smallest domain size. We use three domains so as to compare results with an upcoming study focused on moist dynamics with the same domain setup.

The hodograph of the background directional shear flow is shown in Fig. 1. Seven simulations are performed with three CW hodographs, three CC hodographs, and a unidirectional shear hodograph. The seven simulations are named CW5, CW2.5, CW1.25, unidirectional shear flow (UNIDIR), CC1.25, CC2.5, and CC5. The numbers denote the different amplitudes of the cosine function for meridional wind. They are chosen to have reasonable TCREH as in the real world and also be consistent with ON14 and ON16. In all the simulations, the environmental $u$ component has a cosine shape with easterly winds of $5 \mathrm{~m} \mathrm{~s}^{-1}$ below $850 \mathrm{hPa}$ and a westerly flow of $5 \mathrm{~m} \mathrm{~s}^{-1}$ above $200 \mathrm{hPa}$. Therefore, the deep-layer shear between 200 and $850 \mathrm{hPa}$ in all the simulations are identical to a $10 \mathrm{~m} \mathrm{~s}^{-1}$ westerly shear. The TCREH is positive in the $\mathrm{CW}$ cases and increases from $\mathrm{CW} 1.25$ to CW5. In contrast, the TCREH is negative in the CC cases and decreases from CC1.25 to CC5. As the shear direction is parallel to the environmental flows at all vertical levels, the environmental helicity is zero in the UNIDIR case.

\section{Results}

\section{a. Evolution of overall vortex tilt}

Moist simulations have shown that a continuous precession of convection and an overall vortex tilt toward the upshear region have the potential to reduce the tilt and are thus favorable for TC intensification (Rappin and Nolan 2012; Stevenson et al. 2014; Chen and Gopalakrishnan 2015; Chen et al. 2018). This has also been demonstrated for vortex evolution in a directional shear flow (ON14; ON16). In CW hodographs, the convection can advance more easily toward the upshear region to reduce the overall tilt with subsequent TC intensification. This process involves a feedback from surface latent heat flux and convection (air parcels experiencing larger surface heat flux in a CW hodograph could be more easily ingested

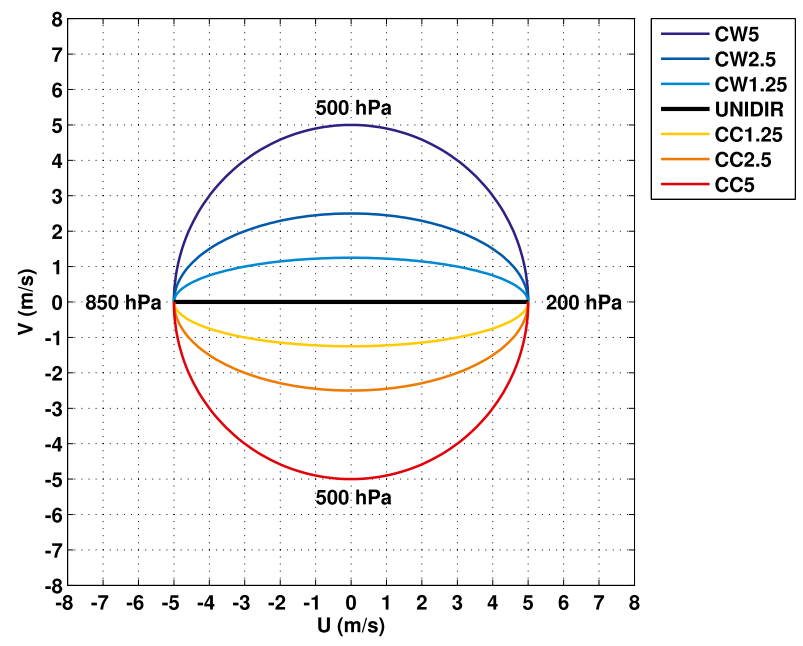

FIG. 1. Hodographs of environmental flow in CW and CC simulations. Red line for CC5, brown line for CC2.5, yellow line for CC1.25, thick black line for UNIDIR, light blue for CW1.25, blue for CW2.5, and dark blue for CW5. The approximate pressure level at which the meridional component of environmental flow achieves its maximum magnitude in the directional shear flows is $500 \mathrm{hPa}$.

into TC core than those in a CC hodograph) and thus highlights the role of moist dynamics (ON16). Here, we show that the difference in overall vortex tilt evolution between the CW and CC hodographs is expected even without the feedback from moist convection and thus has its root in dry dynamics.

In this study, the vortex center at each level is calculated as the vorticity centroid within a $400-\mathrm{km}$ radius of the location of minimum pressure. Directions of vortex tilt are relative to the deep-layer shear direction. Downshear (DS) is $0^{\circ}$, downshear left (DSL) is from $0^{\circ}$ to $90^{\circ}$, left of shear (LS) is $90^{\circ}$, upshear left (USL) is from $90^{\circ}$ to $180^{\circ}$, upshear (US) is $180^{\circ}$, upshear right (USR) is from $180^{\circ}$ to $270^{\circ}$, right of shear (RS) is $270^{\circ}$, and downshear right (DSR) is from $270^{\circ}$ to $360^{\circ}\left(0^{\circ}\right)$. The overall vortex tilt is defined as the tilt between the vortex centers at 8 and $0 \mathrm{~km}$. This is consistent with the vortex tilt defined in ON14 (850 and $300 \mathrm{hPa}$ ) and ON16 (surface and $500 \mathrm{hPa}$ ).

Figure 2 shows a plane view of vortex centers at different height levels (from 2 to $8 \mathrm{~km}$ ) at hours 15 (Fig. 2a) and 35 (Fig. 2b). The initial vortex tilts in different shear hodographs have different directions because of the various meridional winds. At hour 15, the overall vortex tilt is directed toward the downshear-left region in $\mathrm{CW}$ hodographs and toward the downshear (CC1.25) and downshear-right regions (CC2.5 and CC5) in CC hodographs (Fig. 2a). As shown in Fig. 2b, the vortex tilt in all cases precesses cyclonically with a magnitude that increases with time. At hour 35, the vortex tilt in CW5 has precessed close to the left-of-shear region, followed by the tilt in the CW2.5 and CW1.25 cases (Fig. 2b). The vortex 

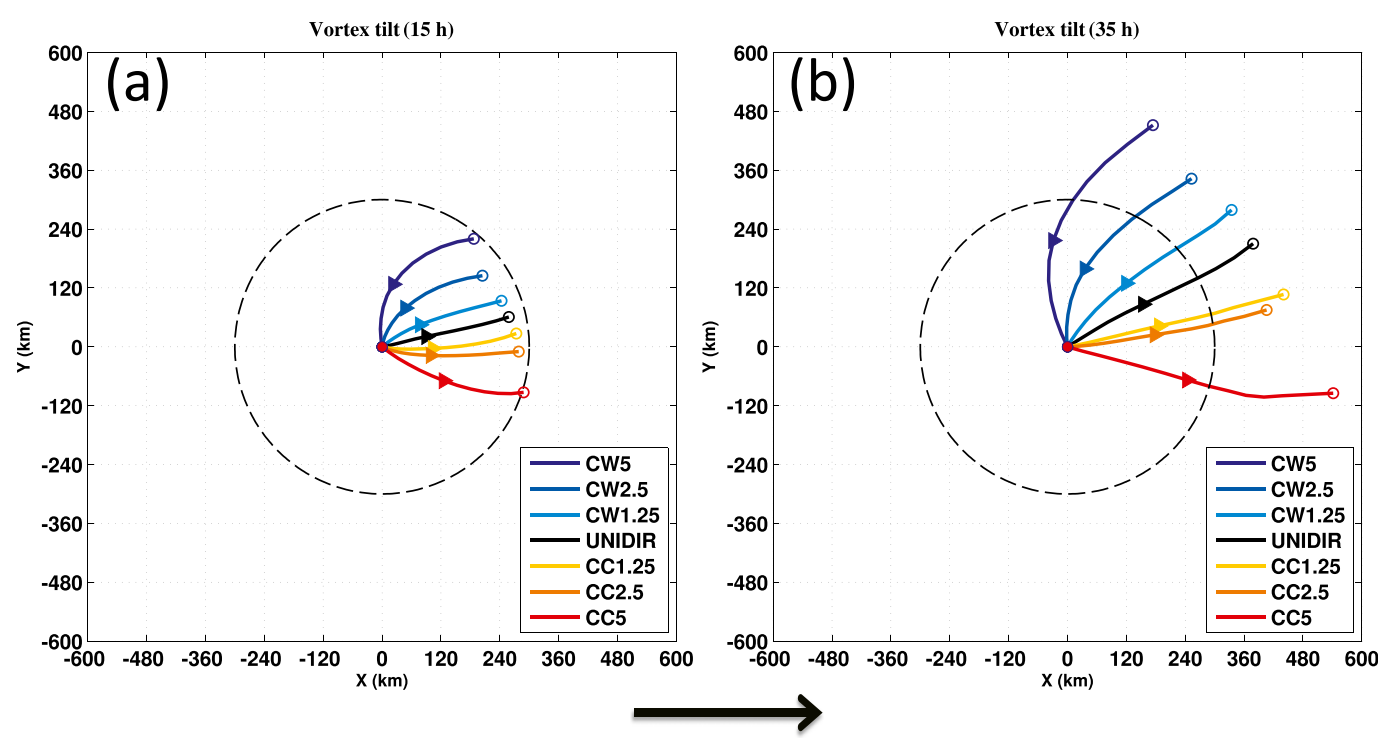

FIG. 2. Plan view of vortex centers at different height levels for all the simulations at hour (a) 15 and (b) 35 . The solid line for each simulation is plotted by connecting the centers from the height of 2 to $8 \mathrm{~km}$ every $1 \mathrm{~km}$. The shaded and hollow circles represent the vortex centers at 2- and 8-km heights, respectively. The shaded triangles represent the center at 5-km height. The centers below $2-\mathrm{km}$ height are not shown since they are close to the center at $2 \mathrm{~km}$. The centers at different levels have been moved to make the centers at 2-km height in all the simulations coincide so that the vortex tilts could be easily compared. Dashed circles in (a) and (b) represent the 300-km radius with respect to the vortex center at 2-km height. Thick black arrow at the bottom of this figure represents the direction of deep-layer shear.

tilt in $\mathrm{CC} 1.25$ and $\mathrm{CC} 2.5$ are both in the downshear-left region, whereas that in CC5 remains in the downshearright region. The vortex tilt is closer to the left-of-shear region at low levels $(0-5 \mathrm{~km})$ and turns toward the downshear-left region at mid- to upper levels $(5-8 \mathrm{~km})$ in CW5 but is closer to the right-of-shear region at low levels $(0-5 \mathrm{~km})$ and turns toward the downshear region at midto upper levels $(5-8 \mathrm{~km})$ in CC5 (Fig. 2b). Therefore, the low-level vortex tilt plays an important role in determining the difference of overall vortex tilt in pure $\mathrm{CW}$ and $\mathrm{CC}$ directional shear flows. This feature is discussed in detail in section $3 \mathrm{c}$.

Figure 3 shows the time evolution of overall vortex tilt $(0-8 \mathrm{~km})$. The overall vortex tilt in all shear flows increases initially and then lessens after around hour 40 . The magnitudes of overall vortex tilt in CW hodographs are smaller than those in $\mathrm{CC}$ hodographs during their steady-state period (after hour 50; Fig. 3a). In CW hodographs, the overall vortex tilt rotates cyclonically from the downshear to the left-of-shear region but from the downshear-right to the downshear region in $\mathrm{CC}$ hodographs (Fig. 3b). Specifically, at steady state, the directions of overall vortex tilt in CW5, CW2.5, and CW1.25 are located in the upshear-left $\left(110^{\circ}\right)$, left-of-shear $\left(90^{\circ}\right)$, and downshear-left $\left(75^{\circ}\right)$ regions, respectively, whereas those in $\mathrm{CC} 1.25, \mathrm{CC} 2.5$, and $\mathrm{CC} 5$ are located in the downshear-left $\left(40^{\circ}\right)$, downshear-left $\left(25^{\circ}\right)$, and downshear $\left(0^{\circ}\right)$ regions, respectively. The vortex tilt direction and magnitude of UNIDIR is between CW and $\mathrm{CC}$ cases. More interestingly, the precession rates of the overall vortex tilt in $\mathrm{CW}$ hodographs are a bit faster than in UNIDIR and CC hodographs. After an initial adjustment $(0-6 \mathrm{~h})$, it takes around $30 \mathrm{~h}$ for the overall vortex tilt to rotate $30^{\circ}$ in $\mathrm{CW}$ hodographs, whereas it takes $40 \mathrm{~h}$ in UNIDIR hodograph and more than $50 \mathrm{~h}$ in CC hodographs (Fig. 3b). For example, it takes about $54 \mathrm{~h}$ (from hour 6 to hour 60) for the overall vortex tilt in CC5 to rotate from $330^{\circ}$ to $0^{\circ}\left(360^{\circ}\right)$. In CW5, the overall vortex tilt only takes $30 \mathrm{~h}$ (from hour 10 to hour 40) to precess $30^{\circ}$ from $60^{\circ}$ to $90^{\circ}$. Thus, the overall vortex tilt in CC5 takes over $20 \mathrm{~h}$ more than that in CW5 to undergo the same precession. Twenty hours is long enough to impact TC evolution considering that rapid intensification is usually defined as significant intensification during a 24-h period.

Vortex tilt evolution can be understood through the interaction between cyclonic circulations at different levels (Jones 1995; Wang and Holland 1996). As the vortex tilt in CC hodographs is closer to the downshear region, the vortex center at high levels is more easily displaced by the environmental flow since the angle between the directions of overall vortex tilt and deeplayer shear in CC hodographs is smaller than that in CW hodographs (Fig. 2). This suggests a stronger interaction 

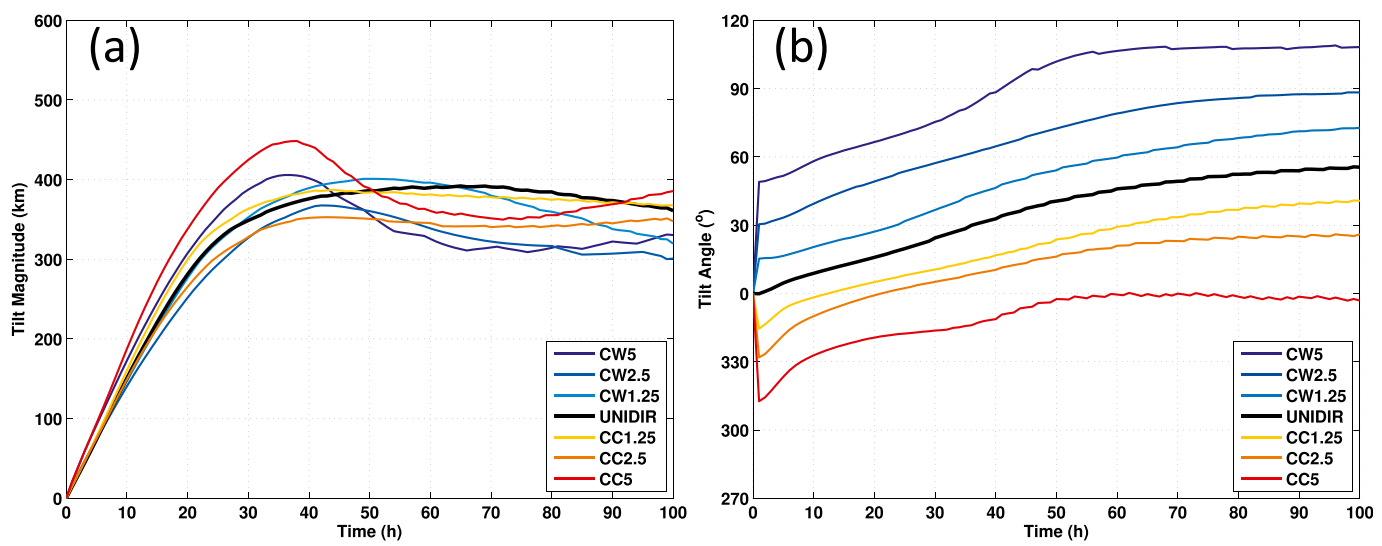

FIG. 3. Time evolution of overall vortex tilt $(0-8 \mathrm{~km})$ (a) magnitude $(\mathrm{km})$ and (b) tilt angle (degree). The tilt angle is calculated counterclockwise from the east $\left(0^{\circ}\right.$ for east, $90^{\circ}$ for north, $180^{\circ}$ for west, and $270^{\circ}$ for south).

between the vortices at lower levels and higher levels in $\mathrm{CW}$ hodographs and thus leads to faster precession rates. As a result, the overall vortex tilts in $\mathrm{CW}$ hodographs are likely to rotate into the upshear region more quickly than do those in CC hodographs, because they have faster precession rates and are closer to the left-ofshear region (Figs. 2 and $3 \mathrm{~b}$ ). The magnitude of vortex tilt is thus easily reduced, resulting in rapid intensification of TCs in moist simulations of CW cases.

\section{b. Kinematic and thermodynamic structure}

In response to directional shear flows, asymmetric structures of TCs will arise at different vertical levels. To identify differences between $\mathrm{CW}$ and CC hodographs, we examine kinematic and thermodynamic structural changes in CW5 and CC5 and compare them with the moist simulations of ON14. The directions used in this analysis are similar to those described in section $3 \mathrm{a}$, with respect to the deep-layer westerly wind shear, unless otherwise noted. The features of relative configurations of these structures with respect to vortex tilt are similar not only in different hodographs but also at different heights and times (not shown) and thus are robust. Here, we choose CW5 and CC5 simply because they have the largest difference in the direction of overall vortex tilt and thus could provide the clearest picture of our points.

Figure 4 shows the asymmetric structures at an altitude of $3 \mathrm{~km}$ at hour 15 . We choose hour 15 for analysis because this time is a suitable one that the moist dynamics are still in the early stage of development and dry dynamics is needed in explaining the features in moist simulations (not shown). At the height of $3 \mathrm{~km}$, the vortex circulation is tilted toward the left-of-shear region in CW5 and toward the downshear-right region in CC5 (Figs. 4a,c). As the environmental flow is southeast wind in $\mathrm{CW} 5$ and northeast wind in $\mathrm{CC} 5$ at low to midlevels, the wind speed is stronger toward the leftof-shear region, with a preference for the downshear-left region in CW5 (Fig. 4a) and the upshear-left region in CC5 (Fig. 4c). In CW5, the cold (warm) anomaly is located in the left-of-shear (right of shear) region (Fig. 4a), and upward (downward) motion is concentrated in the downshear (upshear) region (Fig. 4b). In CC5, the cold (warm) anomaly is located in the downshear-right (upshear left) region (Fig. 4c), and upward (downward) motion is concentrated close to the upshear-right (downshear left) region (Fig. 4d).

Figure 5 shows the kinematic and thermodynamic structures at an altitude of $5 \mathrm{~km}$ at hour 15 . The locations of upward motions and temperature anomalies are a bit different from those at low levels. Because of the directional shear flow, the vortex tilt at this level also changes direction compared to that at low levels. In CW5, the vortex is tilted toward the downshear-left region and closer to the downshear region than that at the height of $3 \mathrm{~km}$. The cold (warm) anomaly moves to the downshear-left (upshear right) region (Fig. 5a), and upward (downward) motion shifts a few degrees clockwise (Fig. 5b) compared to the upward motion at the height of $3 \mathrm{~km}$ (Fig. 4b). In CC5, the cold (warm) anomaly shifts toward the downshear (upshear) region (Fig. 5c) and upward (downward) motion moves counterclockwise to the right-of-shear (left of shear) region (Fig. 5d).

Figures 4 and 5 show that the kinematic and thermodynamic vortex structures have similar configurations with respect to vortex tilt at different vertical levels and are not affected by how the environmental flow changes direction with height. At any given vertical level with a specific vertical tilt, the cold anomaly is located in the downtilt direction and is $180^{\circ}$ out of phase with respect to the warm anomaly. Vertical motion is approximately 

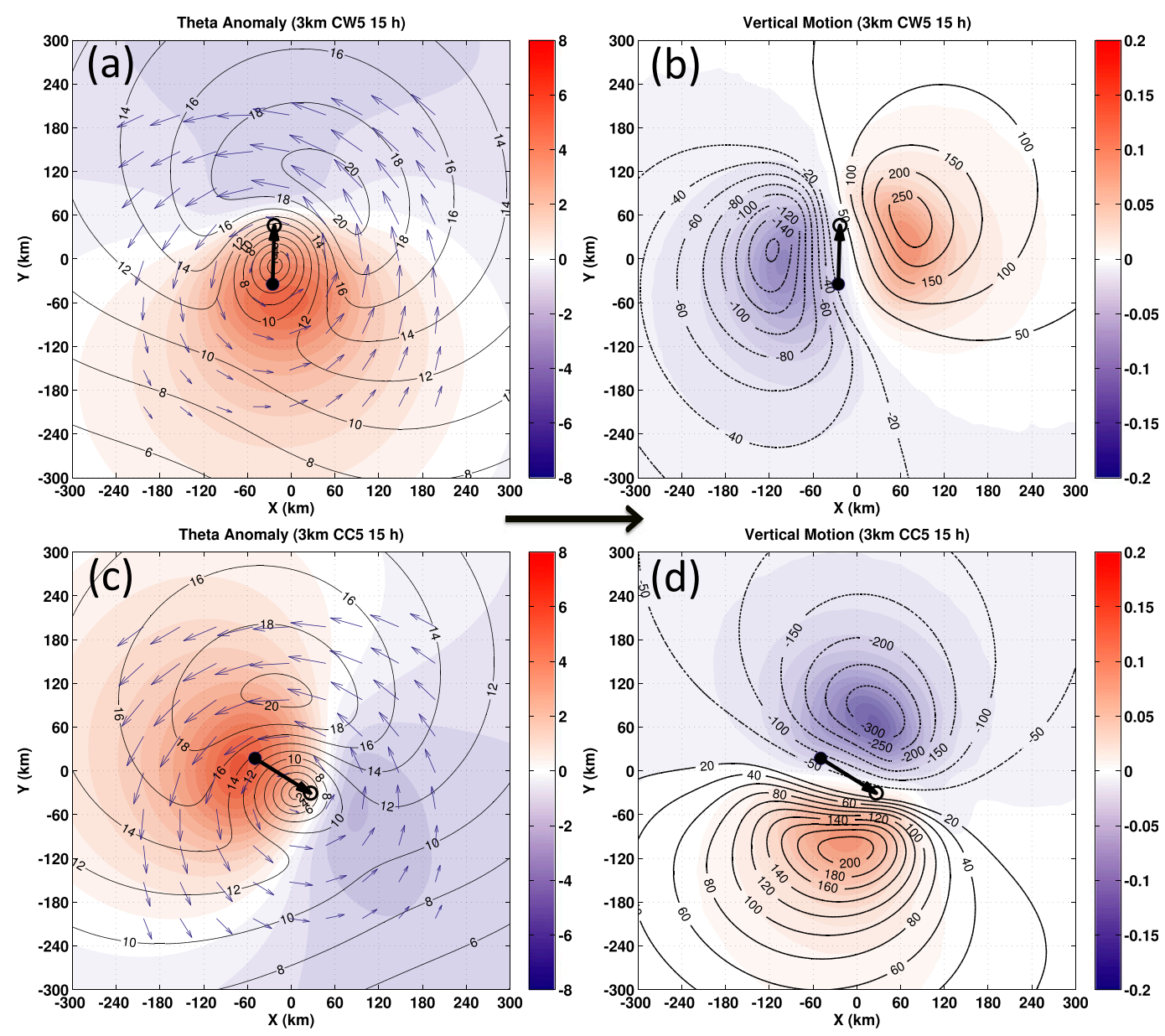

FIG. 4. Kinematic and thermodynamic structures for (a),(b) CW5 and (c),(d) CC5 simulations at 3-km height at hour 15. Horizontal distribution of (a),(c) potential temperature anomaly (K; shaded; blue for cold anomaly and red for warm anomaly), total wind speed $\left(\mathrm{m} \mathrm{s}^{-1}\right.$; black contour), and cyclonic circulation (blue vectors) and

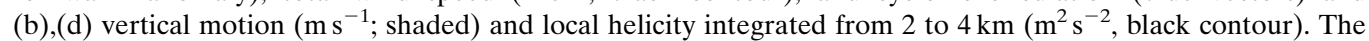
black shaded and hollow circles represent the vortex centers at the height of 2 and $4 \mathrm{~km}$, respectively. The black arrow connecting these two circles represents the vortex tilt between the heights of 2 and $4 \mathrm{~km}$. Temperature anomaly is calculated by subtracting the domain mean from the total potential temperature. The thick black arrow in the middle of this figure represents the direction of deep-layer shear $\left(10 \mathrm{~m} \mathrm{~s}^{-1}\right)$.

$90^{\circ}$ out of phase with the potential temperature anomaly, with upward motion to the right of tilt and downward motion to the left of tilt at each level. When the environmental flow is directional, the upward motion does not always occur in the downshear-left region, as is the case in a unidirectional shear flow because of the distinct vortex tilt direction. This phenomenon was also identified in a numerical study of Typhoon Rananim ( $\mathrm{Li}$ et al. 2008), which shows that the inner-core vertical wind shear and vortex tilt are not unidirectional and the distribution of convection is not consistent with the typical downshear-left pattern. Therefore, at a given vertical level, the vortex tilt is more critical than the deep-layer shear in determining the location of vertical motion.
In both CW5 and CC5, the region of positive (negative) local helicity (integrated near any given level) overlaps with the upward (downward) motion at each level (Figs. 4 and 5). This feature is also present in the other directional shear flow cases (not shown). It indicates that convection is generally located in the region of positive local helicity when moist dynamics are included, consistent with the features in ON14 (their Fig. 9). Evidently, this feature can be well explained by balanced dynamics.

Figure 6 shows a schematic diagram of the relationships between local helicity and vertical motion at a particular level and is applicable to other vertical levels. For simplicity, we assume that the environmental wind shear at this level is westerly and the initial vertically 

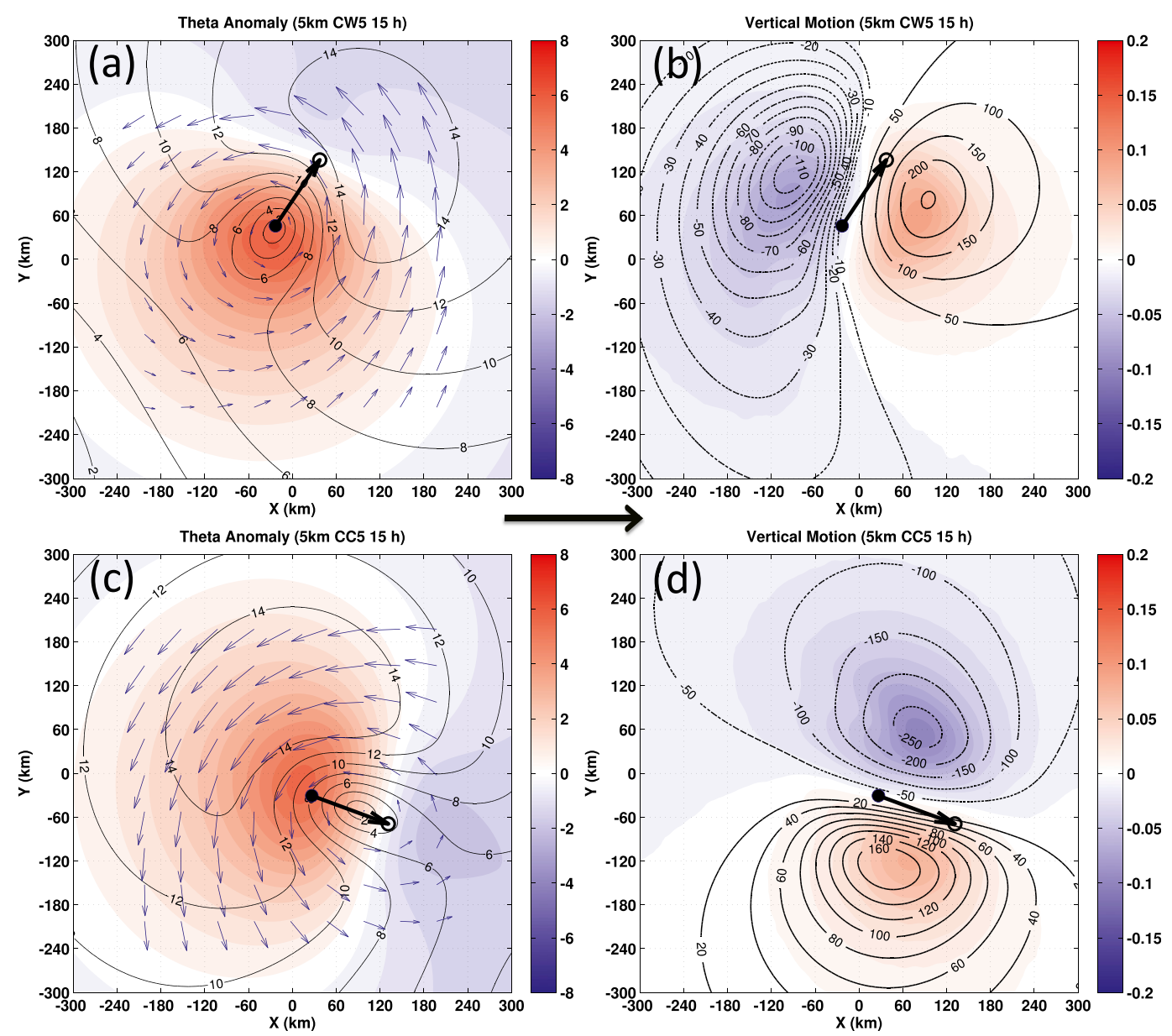

FIG. 5. As in Fig. 4, but for the structures at 5-km height at hour 15. The black shaded and hollow circles represent the vortex centers at the height of 4 and $6 \mathrm{~km}$, respectively. The black arrow connecting these two circles represents the vortex tilt between the heights of 4 and $6 \mathrm{~km}$. The local helicity is integrated from 4 to $6 \mathrm{~km}$.

aligned vortex is tilted from west to east near this level (Fig. 6a). Accordingly, the local wind profile changes from unidirectional to directional shear (Fig. 6b). In the right-of-tilt region, the wind vector rotates clockwise with height, leading to positive local helicity (Fig. 6b). Similarly, negative local helicity occurs in the left-of-tilt region with a local wind vector that rotates counterclockwise with height (Fig. 6b). At the same time, a new local vertical wind shear (black arrows in Fig. 6b) arises because of the tilting of the vortex, directing from the left-of-tilt to right-of-tilt region. To achieve a new thermal wind balance, a temperature gradient should be established from the downtilt to the uptilt region (red arrows in Fig. 6c), indicating that a cold potential temperature anomaly should occur in the downtilt region and a warm potential temperature anomaly in the uptilt region (Fig. 6c). As a result, the isentropic surfaces must be distorted upward at downtilt and downward at uptilt. The flow must be adiabatic as no moist convection is included in the simulation. Therefore, upward motion will be induced to the right-of-tilt region and the downward motion to the left-of-tilt region when the cyclonic circulation flows along the isentropic surfaces. In this way, a new thermal wind balance is achieved, and the upward (downward) motion overlaps with the region of positive (negative) local helicity.

Molinari and Vollaro $(2008,2010)$ proposed that the enhanced in-up-out secondary circulation due to convergence in the downshear in response to the deep-layer shear results in an increase of local helicity. This explains the collocation of convection and large helicity because the primary cyclonic circulation and horizontal vorticity by shear-enhanced secondary circulation are aligned in the same direction in the downshear region. However, this cannot fully explain the diverse distribution of positive local helicity and upward motion in directional shear flows. Here, we propose an alternative explanation. The positive local helicity arises because 


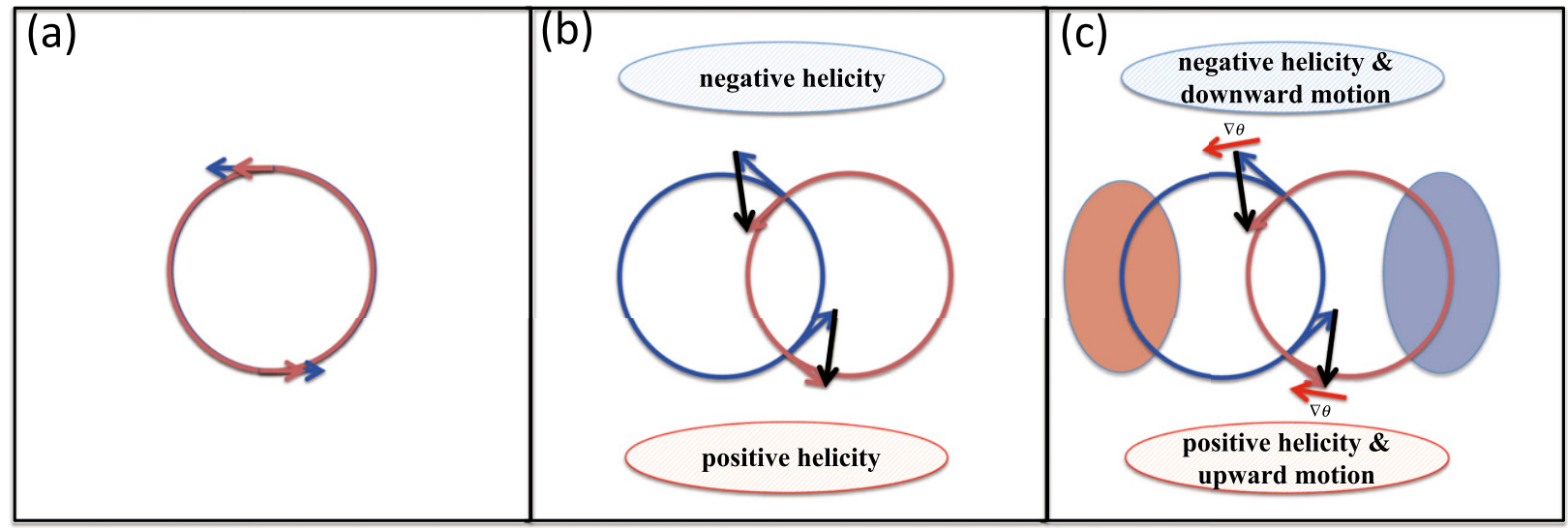

FIG. 6. Schematic diagram for understanding the relationships between the distribution of local helicity and the kinematic and thermodynamic structure change. The panels are arranged in a sequence corresponding to the explanation in section $3 \mathrm{c}$ for (a) vortex configuration before it is tilted; (b) tilted vortex and local vertical wind shear; and (c) distribution of temperature anomaly, vertical motions, and local helicity. Blue and brown circles (thin arrows) represent the low- and upper-level circulation (horizontal wind vectors), respectively. After the vortex is tilted, local directional vertical wind shear occurs, and the black thin arrow represents the local vertical shear vector. The red thin line represents the direction of the temperature gradient in response to the tilt. Shaded ellipses with light blue and red denote the cold and warm anomalies, respectively. The red (blue) patched ellipse represents the region of upward (downward) motion and positive (negative) helicity.

the local horizontal wind is aligned with the horizontal vorticity associated with the local vertical wind shear of tilted vortex rather than the secondary circulation. Balanced dynamics requires that the upward motions should be in phase with positive local helicity, suggesting that the collocation of positive local helicity with upward motions observed in moist simulations (ON14) is not a coincidence but is a natural result of balanced dynamics in response to various vortex tilts in directional shear flows.

The coincidence of local positive (negative) helicity with upward (downward) motion is consistent with previous theoretical studies. Tan and Wu (1994) first found that positive (negative) helicity corresponds to warm (cold)-air advection in cases of thermal wind balance and corresponds to upward (downward) motion in adiabatic flows. Figures 4 and 5 show that the positive local helicity lies between regions of cold and warm anomalies, with the cold anomaly in the downwind region, in both the $\mathrm{CW}$ and $\mathrm{CC}$ cases at different vertical levels. This confirms that the positive local helicity not only coincides with upward motion but is also in phase with warm advection in the framework of balanced dynamics. Hide (2002) derived a relationship between helicity and vertical motion for geostrophic adiabatic flows. This relationship relates helicity $H$ directly with vertical motion $w$ according to $H=N^{2} w /(2 f)$, in which $N$ is the BruntVäisälä frequency and $f$ is the vertical component of planetary rotation. Therefore, it can be expected that positive local helicity will be collocated with upward motion according to dry dynamics, with implications for structures in moist simulations.
Generally speaking, the evolution of vortex tilt, rather than the distribution of local helicity, plays the dominant role in vortex evolution in directional shear flows. This is because vortex structural changes, including the helicity distribution, are simply a result of a balanced adjustment in response to the vortex tilt. Directional shear flows in $\mathrm{CW}$ and $\mathrm{CC}$ hodographs result in quite different behaviors of vortex tilt, thus leading to a diverse distribution of kinematic and thermodynamic structures.

\section{c. Importance of vortex tilt changes with height}

As seen in Figs. 2, 4, and 5, vortex tilt changes direction with height in directional shear flows. This has important implications for understanding the diverse evolution of vortex tilt between the $\mathrm{CW}$ and $\mathrm{CC}$ hodographs. To explain this finding, we compare the results of CW5, UNIDIR, and CC5 at hour 15 (Fig. 7).

Generally, the local wind vector veers in a clockwise (counterclockwise) direction with height to the right (left) of the overall vortex tilt $(0-8 \mathrm{~km})$ in all the simulations (Figs. $7 \mathrm{a}, \mathrm{d}, \mathrm{g}$ ). However, the specific configurations differ. In CW5, there is a region of positive local helicity and mean upward motion $(0-2 \mathrm{~km})$ to the left of the overall vortex tilt (Fig. 7a). This is not the case in UNIDIR and CC5, in which positive local helicity and upward motion are constrained just to the right-of-tilt region (Figs. $7 \mathrm{~d}, \mathrm{~g}$ ). The largest difference arises from the configuration of the low-level vortex tilt relative to the overall vortex tilt. In CW5, the low-level vortex tilt is directed more toward the left-of-shear region than is the overall vortex tilt (Fig. 7b). As a result, positive helicity 

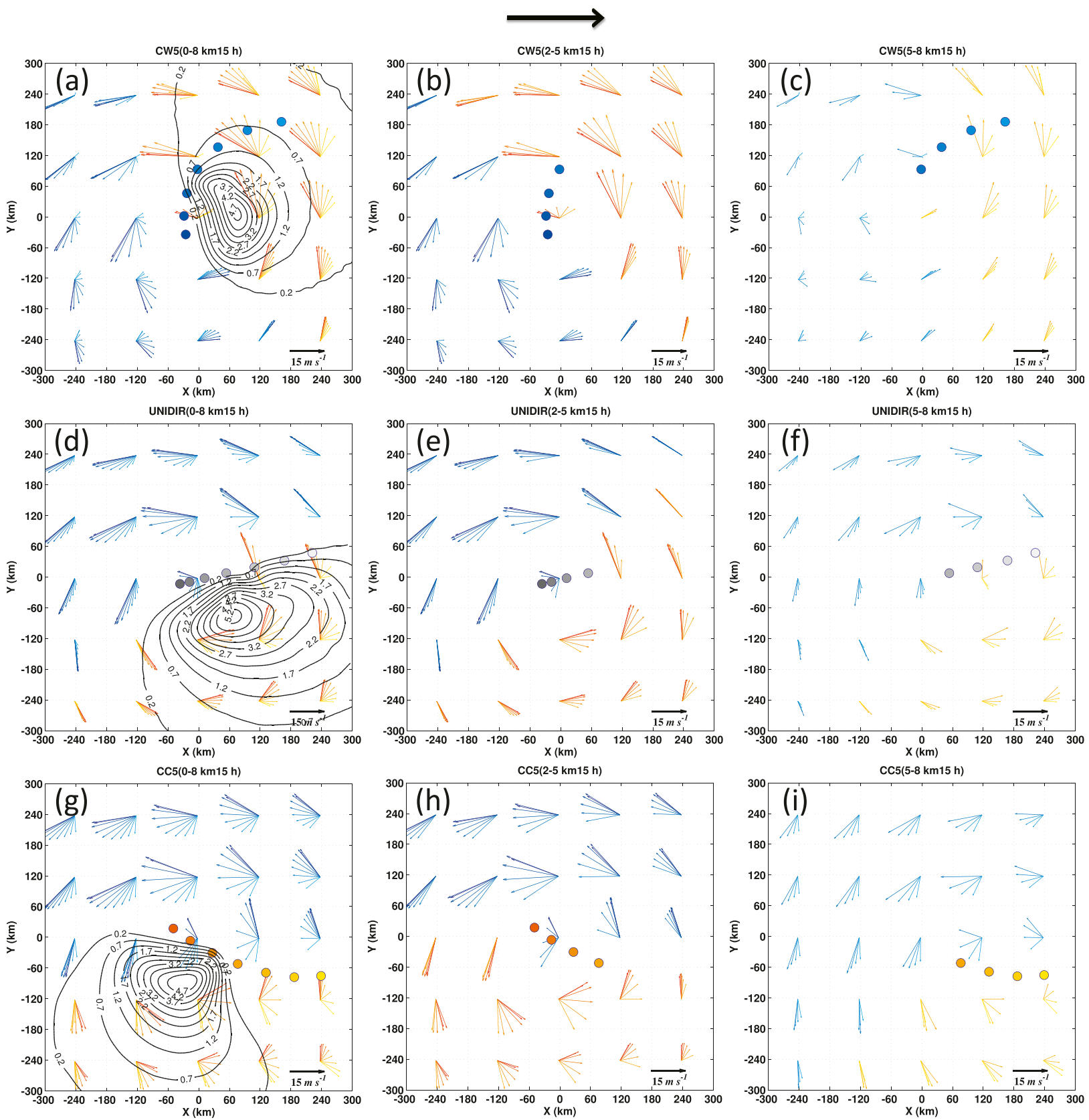

FIG. 7. Local hodographs, upward motions, and vortex centers at different levels in (a)-(c) CW5, (d)-(f) UNIDIR, and (g)-(i) CC5 simulations. (a),(d),(g) The wind vectors are plotted from 2 to $8 \mathrm{~km}$ with 1-km interval (vortex centers below $2 \mathrm{~km}$ coincide with the center at 2-km height); wind vectors with the darkest color (red or blue) are at the surface, while those with lightest color (yellow or light blue) are at 8-km height; warm colors represent positive local helicity, while cold colors represent negative local helicity; the local helicity in (a), (d), and (g) is calculated by integrating the helicity over the $0-8$-km layer; the contour lines represent the $0-2-\mathrm{km}$-averaged upward motions with intervals of $0.5 \mathrm{~cm} \mathrm{~s} \mathrm{~s}^{-1}$; the shaded circles represent the vortex centers from 2- to 8-km height (every $1 \mathrm{~km}$ ) with circles of lighter colors at higher levels. (b),(e),(h) As in (a), (d), and (g), respectively, but the wind vectors and vortex centers are plotted from 2 to $5 \mathrm{~km}$ with 1-km interval, and local helicity is integrated from 2 to $5 \mathrm{~km}$. (c),(f),(i) As in (a), (d), and (g), respectively, but the wind vectors and vortex centers are plotted from 5 to $8 \mathrm{~km}$ with 1 -km interval, and local helicity is integrated from 5 to $8 \mathrm{~km}$. The thick black arrow at the top of this figure represents the direction of deep-layer shear.

and upward motion are located closer to the left-ofshear region at low levels, as explained by the balanced dynamics presented in section $3 \mathrm{~b}$. At mid- to upper levels, the vortex tilt is closer to the downshear region, and thus, positive local helicity and upward motion shift toward the downshear region (Fig. 7c). Therefore, part of the positive local helicity and upward motion at low levels are ahead of the overall vortex tilt in CW5. In 
contrast, in CC5, the vortex tilt at low levels points toward the downshear-right region (Fig. $7 \mathrm{~h}$ ), which is behind the vortex tilt near the downshear region at upper levels (Fig. 7i). In this configuration, positive local helicity and upward motion at low levels are both located in the rear region of the overall vortex tilt (Fig. $7 \mathrm{~g}$ ). In the UNIDIR case, the vortex tilt does not change direction with height, which means that positive local helicity and upward motion will be in the same phase at all levels (Figs. 7e,f). Therefore, positive local helicity and upward motion at low levels are just to the right of the overall vortex tilt (Fig. 7d). Note that the low-level upward motions are much smaller than those at midlevels. This is because there is no obvious vortex tilt below $2-\mathrm{km}$ height and the response to low-level tilt is small. However, the low-level upward motions will be amplified once the moist convection is included in the simulations.

The different configurations of positive local helicity and upward motion with respect to the overall vortex tilt may be potentially responsible for the diverse vortex evolution in directional shear flows, once moist dynamics are considered. In $\mathrm{CW}$ hodographs, the convection is more easily triggered and maintained ahead of the overall vortex tilt and thus would favor its continuous precession. In CC hodographs, the convection is generally concentrated behind the overall vortex tilt and slows the precession. As a result, the vortex tilt and convection in CW hodographs are more likely to advance into the upshear region than those in CC hodographs, thus leading to TC intensification. Therefore, vortex tilt that varies with height, particularly that related to the relative configuration of the vortex tilt at low levels with respect to the overall vortex tilt, may have a large impact on the distribution of convection and thus the intensification behavior of TCs in pure $\mathrm{CW}$ or $\mathrm{CC}$ directional shear flows.

\section{Discussion}

In moist simulations, the various intensification rates of TCs in the CW and CC hodographs are attributed to differences in vortex structure (N11; ON14; ON16). One may conclude that feedbacks from convection play a dominant role, because they depend on moist dynamics. We have shown in this study that these differences have their roots in dry dynamics. The distinct features of a vortex structure in directional shear flows can be well explained by balanced dynamics in the absence of moist convection. Possible processes that lead to observed differences in moist simulations are as follows. First, the vortex is tilted by the directional shear flows, and the manner in which vortex tilt changes with height depends on the details of the hodograph. The evolution of the overall vortex tilt includes contributions from the interaction between cyclonic circulations at different vertical levels. The thermal wind balance within the initial vertically aligned vortex breaks up following the beginning of vortex tilt. In response to the vortex tilt, kinematic and thermodynamic structural changes of a TC arise to achieve a new balanced state. The positive (negative) local helicity coincides with upward (downward) motion and is in the region of warm (cold) advection. The distribution of these structural changes relative to the deep-layer shear differs considerably because of the differing overall vortex tilt in $\mathrm{CW}$ and $\mathrm{CC}$ hodographs. These differences further affect the evolution of overall vortex tilt and thus the TC intensification rate once the feedback from convection is included. The perspective of helicity from ON14 provides new insight, but it is a part of the balanced dynamics. Thus, dry dynamics controls the initial vortex tilt evolution and structural changes of a TC and is a major component of TC evolution in directional shear flows.

The differences of overall vortex tilt evolution in directional shear flows could also be understood through vortex Rossby wave (VRW) dynamics (Reasor 2000; Reasor and Montgomery 2001; Schecter et al. 2002; Schecter and Montgomery 2003; Reasor et al. 2004). The VRWs will be excited once the initially vertically aligned vortex is tilted by the vertical wind shear. The evolution of vortex tilt can be viewed as a superposition of the sheared VRWs and a quasi mode (Reasor and Montgomery 2001; Reasor et al. 2004). In this study, the tilted vortex supports a quasi mode (no obvious sheared VRWs exist; not shown) and thus has a clear precession, as shown in Fig. 3b. A quasi mode will be damped through a resonance with the fluid rotation at the critical radius, which is defined as the location where the precession rate equals the angular rotation rate of the mean vortex (Schecter et al. 2002). The damping rate is proportional to the radial gradient of potential vorticity at the critical radius (Schecter and Montgomery 2003). In the $\mathrm{CW}$ cases, the critical radius of the tilted vortex is smaller than that in the $\mathrm{CC}$ cases because of the faster precession rate. As a result, the damping rate of vortex tilt in the $\mathrm{CW}$ cases is larger because of the larger magnitude of the negative radial gradient of potential vorticity at the critical radius. This explains the smaller vortex tilt at steady state (after hour 50) in the CW cases. In a unidirectional shear flow, the time-invariant forcing results in a forced damped oscillator with a downshear-left configuration near steady state (Reasor et al. 2004). In a directional shear flow, the optimal configuration of the vortex tilt at steady state will differ depending on the structure of the vertical wind shear. This can be understood by adding an additional time-invariant forcing 

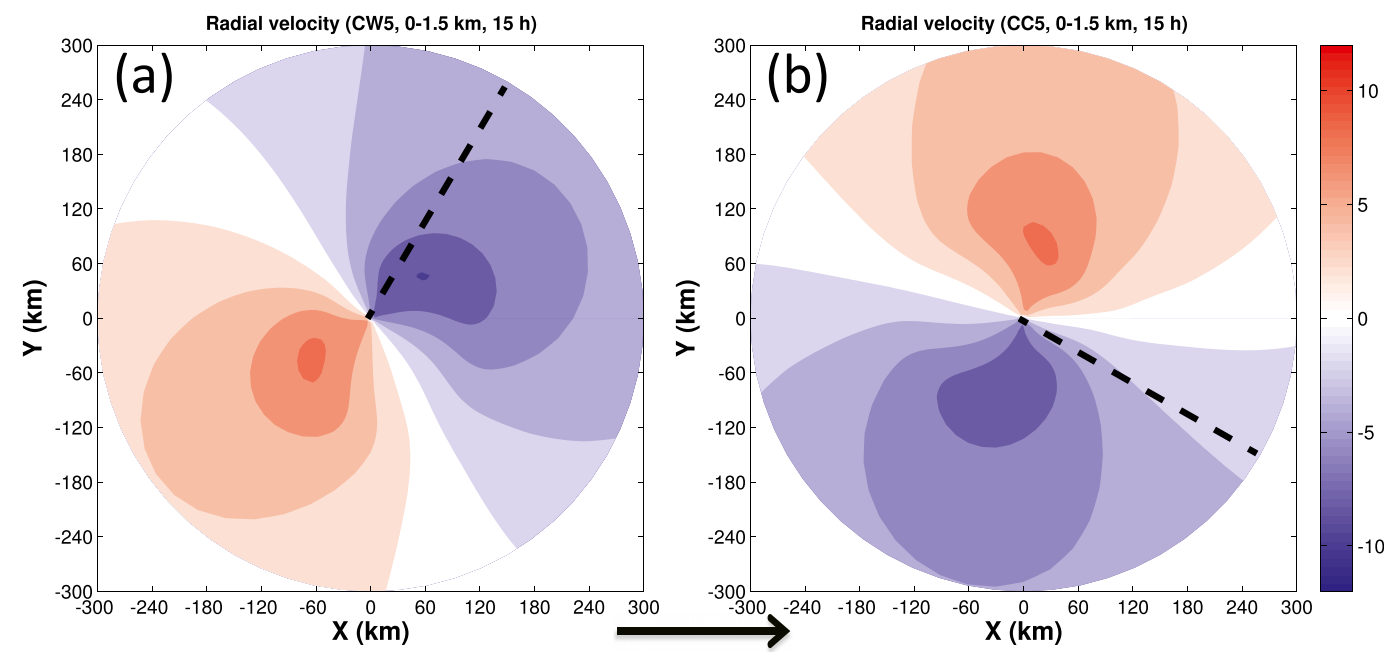

FIG. 8. Plan view of asymmetric structures of radial flows $\left(\mathrm{m} \mathrm{s}^{-1}\right)$ in the boundary layer $(0-1.5 \mathrm{~km})$ at hour 15 : (a) CW5 and (b) CC5. The thick black arrow at the bottom of this figure represents the direction of deep-layer shear. Black dashed lines in (a) and (b) represent the direction of the overall vortex tilt in CW5 and CC5 at hour 15 .

term that varies with height on the right-hand side of Eq. (20) in Reasor et al. (2004). This height-varying forcing has opposite signs for CW and CC hodographs and thus will result in different overall tilt directions in simulations over long time periods: close to the left-of-shear region in $\mathrm{CW}$ cases and close to the downshear region in $\mathrm{CC}$ cases.

It should be noted that other processes, such as boundary layer processes, may also contribute to vortex evolution in directional shear flows. Friction within the boundary layer turns low-level flows inward to increase the local helicity and introduce unbalanced processes. To demonstrate the balanced dynamics clearly as much as possible, we do not discuss the effects of boundary layer friction in this study, and the planetary boundary layer scheme is not turned on in our simulations. However, the asymmetric structure of radial inflows within the boundary layer may also affect the distribution of helicity and convection. For CW hodographs, the environmental flows are generally directed toward the north from 850 to $200 \mathrm{hPa}$, resulting in a northward movement of the vortex. In contrast, CC hodographs lead to southward vortex movement. Diverse vortex movement results in considerably different radial inflow structures. For example, radial inflows are mainly concentrated in the downshear-left region in CW5 (Fig. 8a) but are located in the right-of-shear region in CC5 (Fig. 8b). Boundary layer inflows in CW5 might tend to trigger convection and increase the local helicity ahead of overall vortex tilt, favoring continuous precession. However, boundary layer inflows in $\mathrm{CC} 5$ tend to maintain the convection in the right-of-shear region behind the overall vortex tilt and thus slow the precession.
Though our study only examines the weak vortex, the results could still provide useful insights for the understanding of the evolution of mature vortices in the directional shear flows. While mature storms have more ability to survive in the vertical wind shear, the way in which the vortex is tilted at different levels will be qualitatively the same as the weak vortices. The overall vortex tilt in $\mathrm{CW}$ hodographs will generally direct toward the left-of-shear region and that in CC hodographs will be in the downshear region. The low-level vortex tilt is ahead (behind) of the overall vortex tilt in CW (CC) cases. Balanced dynamics will still result in similar relationships between upward motions and positive local helicity and their configurations relative to the overall vortex tilt. Therefore, the difference of vortex tilt evolution and favorable locations of convection in mature storms embedded in CW and CC hodographs will be qualitatively consistent with that for weak TCs in this study.

\section{Summary}

Using idealized dry simulations, this study finds that the evolution of vortex tilt and the asymmetric distribution of kinematic and thermodynamic structures of vortex in directional shear flows are highly variable and depend on how environmental flows rotate with height, even with identical deep-layer vertical wind shear. The evolution of vortex tilt is controlled primarily by the interaction between cyclonic circulations at different levels and the environmental flow. It is found that the overall vortex tilt precesses cyclonically with larger 
precession rates in $\mathrm{CW}$ hodographs than in $\mathrm{CC}$ hodographs. The height-dependent vortex tilt gradually achieves a steady state in the left-of-shear region at low levels and the downshear-left region at mid- to upper levels in CW hodographs, whereas that in CC hodographs reaches a steady direction in the downshearright region at low levels and the downshear region at mid- to upper levels (Fig. 9). Because the overall vortex tilt is closer to the upshear region in CW hodographs, it is more likely to be reduced and thus result in TC intensification than in $\mathrm{CC}$ hodographs when moist convection is considered.

The kinematic and thermodynamic structures of a vortex have a fixed configuration relative to vortex tilt at all vertical levels in both the $\mathrm{CW}$ and $\mathrm{CC}$ cases. A cold anomaly occurs in the downtilt region with upward motion located $90^{\circ}$ to the right of vortex tilt and a warm anomaly in the uptilt region with downward motion $90^{\circ}$ to the left of vortex tilt. This is similar to the situation in unidirectional shear flows as discussed by Jones (1995), but the configuration is more complicated. Because the overall vortex tilt has a different direction and precession rate, upward motion has a diverse distribution in the $\mathrm{CW}$ and CC cases. In CW hodographs, upward motion generally occurs from the downshear to downshear-left region, whereas in CC hodographs, it rises from the rightof-shear to downshear-right region. This provides a possible explanation for the inconsistencies of the typical downshear-left pattern of a shear-induced inner-core distribution of convection in observations and numerical simulations.

As vortex tilt gradually changes direction with height in directional shear flow, the kinematic and thermodynamic structures also change but retain their relative configuration with respect to the vortex tilt direction at each vertical level. Moreover, the varying vortex tilt with height has an important implication for the diverse TC evolution in directional shear flows. Figure 9 has a brief demonstration of how it works. In CW hodographs, because the low-level vortex tilt is closer to the left-ofshear region than the overall vortex tilt, there is a region with positive local helicity and upward motion at low levels ahead of the overall vortex tilt (Fig. 9). This configuration is potentially favorable for the continuous precession of convection and overall vortex tilt into the upshear region if moist dynamics are included. In contrast, in CC cases, the low-level vortex tilt, as well as the positive local helicity and upward motion at low levels, is behind the overall vortex tilt and thus does not promote vortex tilt precession (Fig. 9).

In addition, positive (negative) local helicity is in phase with upward (downward) motion. This explains the overlap between positive local helicity and convection in

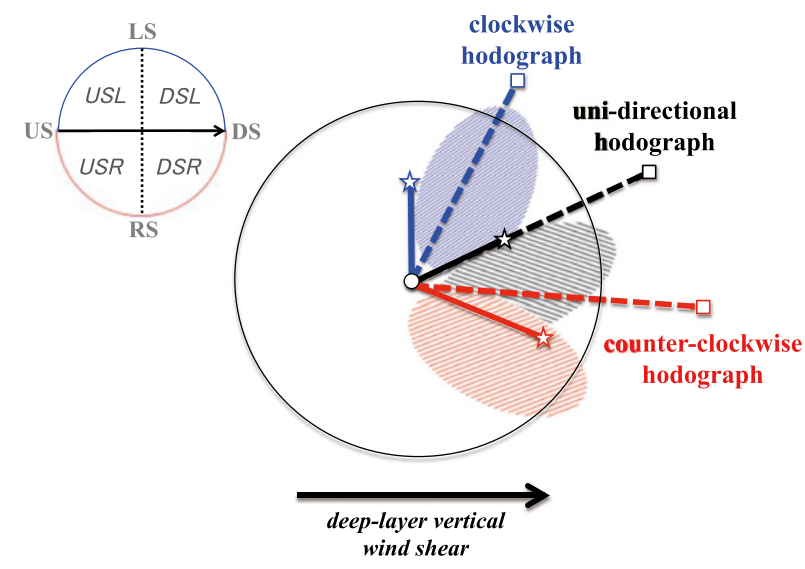

FIG. 9. Schematic diagram showing the configuration of lowlevel vortex tilt, overall vortex tilt, and low-level upward motions in directional and unidirectional shear flows. The largest thin black circle represents the TC's inner-core region. The smallest black circle at the center of the inner-core region represents the vortex center at the surface. In the UNIDIR, the black star and square represent the vortex centers at the mid- and upper levels, respectively; the thick black solid line and dashed line represent the low-level and overall vortex tilt, respectively; the black patched area represents the region with upward motions at low levels. In the directional shear flow with the clockwise hodograph, the blue star and square represent the vortex centers at the midand upper levels, respectively; the thick blue solid line and dashed line represent the low-level and overall vortex tilt, respectively; the blue patched area represents the region with upward motions at low levels. In the directional shear flow with the counterclockwise hodograph, the red star and square represent the vortex centers at the mid- and upper levels, respectively; the red solid line and dashed line represent the low-level and overall vortex tilt, respectively; the red patched area represents the region with upward motions at low levels. The upper-left circle represents the hodographs for UNIDIR (small black arrow) and directional (blue semicircle for clockwise and red semicircle for counterclockwise) shear flow. The black dashed line within this circle is plotted to help identify the azimuth position relative to the deeplayer shear: DS: downshear; RS: right of shear; US: upshear; LS: left of shear; DSL: downshear left; DSR: downshear right; USR: upshear right; USL: upshear left. The thick black arrow at the bottom of the figure represents the deep-layer shear. The lowlevel vortex tilt is ahead (behind) the overall vortex tilt in $\mathrm{CW}$ (CC) hodographs, while it is aligned with the overall vortex tilt in UNIDIR. These differences set up the differences of favorable locations of convection and the abilities of continuous precession in the directional shear flows.

moist simulations (ON14). This coincidence is not only the result of shear-enhanced secondary circulation, as noted in Molinari and Vollaro (2008, 2010), but also comes from a balance adjustment because of the vortex tilt. This is consistent with theoretical studies of helicity dynamics suggesting that helicity is closely related with temperature advection (Tan and $\mathrm{Wu} 1994$ ) and upward motion (Hide 2002).

In moist simulations of TCs in directional shear flows (N11; ON14; ON16), the diverse evolution of 
TC intensity was found to be associated with differences in the distributions of inner-core convection and feedback from the surface heat flux in directional shear flows. Although moist processes will help to damp the overall vortex tilt and new mechanisms might take effect, the dry balanced dynamics may still regulate structural changes under moist conditions at early stages and provide the basis for moist feedback to work subsequently. This regulation determines the initial evolution of vortex tilt and inner-core convection and thus may be responsible for subsequent TC intensity changes. For example, CW hodographs favor a faster precession of vortex tilt and result in an equilibrium state with a vortex tilt closer to the upshear region than is seen in CC hodographs. When coupled with latent heating from moist convection, this may contribute to the faster propagation of convective clusters in $\mathrm{CW}$ hodographs and result in more rapid TC intensification even without the feedback from surface heat flux. A detailed investigation of the coupling between moist and dry dynamics in directional shear flows will be the focus of an upcoming study.

Acknowledgments. We thank David S. Nolan and Matthew J. Onderlinde for their suggestions on the implementation of the PDS method in the WRF Model. The first author also thanks Dr. Christopher E. Holloway for discussion. The authors also thank three anonymous reviewers for their critical comments and constructive suggestions that significantly improve the manuscript. This work was jointly supported by the National Key R\&D Program of China under Grant 2017YFC1501601, the National Natural Science Foundation of China (41505044, 41575053, 41675053), National Key Project for Basic Research (973 Project) under Grants 2015CB452803 and 2013CB834102, and Joint Research Project of State Key Laboratory of Severe Weather of Chinese Academy of Meteorological Sciences.

\section{REFERENCES}

Black, M. L., J. F. Gamache, F. D. Marks, C. E. Samsury, and H. E. Willoughby, 2002: Eastern Pacific Hurricanes Jimena of 1991 and Olivia of 1994: The effect of vertical shear on structure and intensity. Mon. Wea. Rev., 130, 2291-2312, https://doi.org/ 10.1175/1520-0493(2002)130<2291:EPHJOA > 2.0.CO;2.

Chen, H., and S. G. Gopalakrishnan, 2015: A study on the asymmetric rapid intensification of Hurricane Earl (2010) using the HWRF system. J. Atmos. Sci., 72, 531-550, https://doi.org/ 10.1175/JAS-D-14-0097.1.

Chen, X., Y. Wang, J. Fang, and M. Xue, 2018: A numerical study on rapid intensification of Typhoon Vicente (2012) in the South China Sea. Part II: Roles of inner-core processes. J. Atmos. Sci. 75, 235-255, https://doi.org/10.1175/JAS-D-17-0129.1.

Corbosiero, K. L., and J. Molinari, 2003: The relationship between storm motion, vertical wind shear, and convective asymmetries in tropical cyclones. J. Atmos. Sci., 60, 366-376, https://doi.org/ 10.1175/1520-0469(2003)060<0366:TRBSMV>2.0.CO;2.

DeHart, J. C., R. A. Houze Jr., and R. F. Rogers, 2014: Quadrant distribution of tropical cyclone inner-core kinematics in relation to environmental shear. J. Atmos. Sci., 71, 2713-2732, https://doi.org/10.1175/JAS-D-13-0298.1.

DeMaria, M., 1996: The effect of vertical shear on tropical cyclone intensity change. J. Atmos. Sci., 53, 2076-2088, https://doi.org/ 10.1175/1520-0469(1996)053<2076:TEOVSO >2.0.CO;2.

Dunion, J. P., 2011: Rewriting the climatology of the tropical North Atlantic and Caribbean Sea atmosphere. J. Climate, 24, 893908, https://doi.org/10.1175/2010JCLI3496.1.

Finocchio, P. M., S. J. Majumdar, D. S. Nolan, and M. Iskandarani, 2016: Idealized tropical cyclone responses to the height and depth of environmental vertical wind shear. Mon. Wea. Rev., 144, 2155-2175, https://doi.org/10.1175/MWR-D-15-0320.1.

Frank, W. M., and E. A. Ritchie, 2001: Effects of vertical wind shear on the intensity and structure of numerically simulated hurricanes. Mon. Wea. Rev., 129, 2249-2269, https://doi.org/ 10.1175/1520-0493(2001)129<2249:EOVWSO > 2.0.CO;2.

Gray, W. M., 1968: Global view of the origin of tropical disturbances and storms. Mon. Wea. Rev., 96, 669-700, https://doi.org/ 10.1175/1520-0493(1968)096<0669:GVOTOO>2.0.CO;2.

Gu, J.-F., Z.-M. Tan, and X. Qiu, 2015: Effects of vertical wind shear on inner-core thermodynamics of an idealized simulated tropical cyclone. J. Atmos. Sci., 72, 511-530, https://doi.org/ 10.1175/JAS-D-14-0050.1.

__ —_, and ——, 2016: Quadrant-dependent evolution of low-level tangential wind of a tropical cyclone in the shear flow. J. Atmos. Sci., 73, 1159-1177, https://doi.org/10.1175/ JAS-D-15-0165.1.

Hide, R., 2002: Helicity, superhelicity and weighted relative potential vorticity: Useful diagnostic pseudoscalars? Quart. J. Roy. Meteor. Soc., 128, 1759-1762, https://doi.org/10.1002/ qj.200212858318.

Jones, S. C., 1995: The evolution of vortices in vertical shear. I: Initially barotropic vortices. Quart. J. Roy. Meteor. Soc., 121, 821-851, https://doi.org/10.1002/qj.49712152406.

Li, Q., Y. Duan, H. Yu, and G. Fu, 2008: A high-resolution simulation of Typhoon Rananim (2004) with MM5. Part I: Model verification, inner-core shear, and asymmetric convection. Mon. Wea. Rev., 136, 2488-2506, https://doi.org/ 10.1175/2007MWR2159.1.

Molinari, J., and D. Vollaro, 2008: Extreme helicity and intense convective towers in Hurricane Bonnie. Mon. Wea. Rev., 136, 4355-4372, https://doi.org/10.1175/2008MWR2423.1.

— and - 2010: Distribution of helicity, CAPE, and shear in tropical cyclones. J. Atmos. Sci., 67, 274-284, https://doi.org/ 10.1175/2009JAS3090.1.

Nolan, D. S., 2011: Evaluating environmental favorableness for tropical cyclone development with the method of pointdownscaling. J. Adv. Model. Earth Syst., 3, M08001, https://doi.org/ 10.1029/2011MS000063.

Onderlinde, M. J., and D. S. Nolan, 2014: Environmental helicity and its effects on development and intensification of tropical cyclones. J. Atmos. Sci., 71, 4308-4320, https://doi.org/10.1175/ JAS-D-14-0085.1.

- , and - , 2016: Tropical cyclone-relative environmental helicity and the pathways to intensification in shear. J. Atmos. Sci., 73, 869-890, https://doi.org/10.1175/JAS-D-15-0261.1.

Rappin, E. D., and D. S. Nolan, 2012: The effects of vertical shear orientation on tropical cyclogenesis. Quart. J. Roy. Meteor. Soc., 138, 1035-1054, https://doi.org/10.1002/qj.977. 
Reasor, P. D., 2000: Horizontal vorticity redistribution and vortex alignment in developing and mature tropical cyclones. Ph.D. dissertation, Colorado State University, $166 \mathrm{pp}$.

—_, and M. T. Montgomery, 2001: Three-dimensional alignment and corotation of weak, TC-like vortices via linear vortex Rossby waves. J. Atmos. Sci., 58, 2306-2330, https://doi.org/ 10.1175/1520-0469(2001)058<2306:TDAACO >2.0.CO;2.

, - — and L. D. Grasso, 2004: A new look at the problem of tropical cyclones in vertical wind shear flow: Vortex resiliency. J. Atmos. Sci., 61, 3-22, https://doi.org/10.1175/15200469(2004)061<0003:ANLATP>2.0.CO;2.

_, R. Rogers, and S. Lorsolo, 2013: Environmental flow impacts on tropical cyclone structure diagnosed from airborne Doppler radar composites. Mon. Wea. Rev., 141, 2949-2969, https:// doi.org/10.1175/MWR-D-12-00334.1.

Riemer, M., M. T. Montgomery, and M. Nicholls, 2010: A new paradigm for intensity modification of tropical cyclones: Thermodynamic impact of vertical wind shear on the inflow layer. Atmos. Chem. Phys., 10, 3163-3188, https://doi.org/ 10.5194/acp-10-3163-2010.

Schecter, D. A., and M. T. Montgomery, 2003: On the symmetrization rate of an intense geophysical vortex. Dyn. Atmos. Oceans, 37, 55-88, https://doi.org/10.1016/S0377-0265(03)00015-0.

—_ —, and P. D. Reasor, 2002: A theory for the vertical alignment of a quasi-geostrophic vortex. J. Atmos. Sci., 59, 150-168, https://doi.org/10.1175/1520-0469(2002)059<0150: ATFTVA $>2.0 . \mathrm{CO} ; 2$.

Simpson, R., and R. Riehl, 1958: Mid-tropospheric ventilation as a constraint on hurricane development and maintenance. Preprints, Tech. Conf. on Hurricanes, Miami Beach, FL, Amer. Meteor. Soc., D4-1-D4-10.
Skamarock, W. C., and Coauthors, 2008: A description of the Advanced Research WRF version 3. NCAR Tech. Note NCAR/ TN-475+STR, 113 pp., https://doi.org/10.5065/D68S4MVH.

Stevenson, S. N., K. L. Corbosiero, and J. Molinari, 2014: The convective evolution and rapid intensification of Hurricane Earl (2010). Mon. Wea. Rev., 142, 4364-4380, https://doi.org/ 10.1175/MWR-D-14-00078.1.

Tan, Z.-M., and R. Wu, 1994: Helicity dynamics of atmospheric flow. Adv. Atmos. Sci. 11, 175-188, https://doi.org/10.1007/BF02666544.

Tang, B., and K. Emanuel, 2010: Midlevel ventilation's constraint on tropical cyclone intensity. J. Atmos. Sci., 67, 1817-1830, https://doi.org/10.1175/2010JAS3318.1.

Tuleya, R. E., and Y. Kurihara, 1981: A numerical study on the effects of environmental flow on tropical storm genesis. Mon. Wea. Rev., 109, 2487-2506, https://doi.org/10.1175/ 1520-0493(1981)109<2487:ANSOTE > 2.0.CO;2.

Wang, Y., and G. J. Holland, 1996: Tropical cyclone motion and evolution in vertical shear. J. Atmos. Sci., 53, 3313-3332, https:// doi.org/10.1175/1520-0469(1996)053<3313:TCMAEI >2.0.CO;2.

_ , Y. Rao, Z.-M. Tan, and D. Schönemann, 2015: A statistical analysis of the effects of vertical wind shear on tropical cyclone intensity change over the western North Pacific. Mon. Wea. Rev., 143, 3434-3453, https://doi.org/10.1175/MWR-D-15-0049.1.

$\mathrm{Wu}, \mathrm{L}$., and S. Braun, 2004: Effects of environmentally induced asymmetries on hurricane intensity: A numerical study. J. Atmos. Sci., 61, 3065-3081, https://doi.org/10.1175/JAS-3343.1.

Zhang, J. A., R. F. Rogers, P. D. Reasor, E. W. Uhlhorn, and F. D. Marks Jr., 2013: Asymmetric hurricane boundary layer structure from dropsonde composites in relation to the environmental vertical wind shear. Mon. Wea. Rev., 141, 39683984, https://doi.org/10.1175/MWR-D-12-00335.1. 